\title{
Investigation of a plasma-target interaction through electric field characterization examining surface and volume charge contributions: modeling and experiment
}

\author{
Citation for published version (APA): \\ Viegas, P., Slikboer, E., Obrusnik, A., Bonaventura, Z., Sobota, A., Garcia-Caurel, E., Guaitella, O., \& Bourdon, \\ A. (2018). Investigation of a plasma-target interaction through electric field characterization examining surface \\ and volume charge contributions: modeling and experiment. Plasma Sources Science and Technology, 27(9), \\ [094002]. https://doi.org/10.1088/1361-6595/aadcc0
}

\section{Document license: \\ TAVERNE}

DOI:

10.1088/1361-6595/aadcc0

Document status and date:

Published: 14/09/2018

\section{Document Version:}

Publisher's PDF, also known as Version of Record (includes final page, issue and volume numbers)

\section{Please check the document version of this publication:}

- A submitted manuscript is the version of the article upon submission and before peer-review. There can be important differences between the submitted version and the official published version of record. People interested in the research are advised to contact the author for the final version of the publication, or visit the $\mathrm{DOI}$ to the publisher's website.

- The final author version and the galley proof are versions of the publication after peer review.

- The final published version features the final layout of the paper including the volume, issue and page numbers.

Link to publication

\footnotetext{
General rights

- You may freely distribute the URL identifying the publication in the public portal. follow below link for the End User Agreement:

www.tue.nl/taverne

\section{Take down policy}

If you believe that this document breaches copyright please contact us at:

openaccess@tue.nl

providing details and we will investigate your claim.
}

Copyright and moral rights for the publications made accessible in the public portal are retained by the authors and/or other copyright owners and it is a condition of accessing publications that users recognise and abide by the legal requirements associated with these rights.

- Users may download and print one copy of any publication from the public portal for the purpose of private study or research.

- You may not further distribute the material or use it for any profit-making activity or commercial gain

If the publication is distributed under the terms of Article 25fa of the Dutch Copyright Act, indicated by the "Taverne" license above, please 


\section{PAPER}

Investigation of a plasma-target interaction through electric field characterization examining surface and volume charge contributions: modeling and experiment

To cite this article: Pedro Viegas et al 2018 Plasma Sources Sci. Technol. 27094002

View the article online for updates and enhancements.

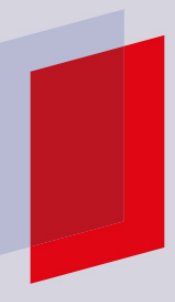

\section{IOP ebooks}

Bringing you innovative digital publishing with leading voices

to create your essential collection of books in STEM research.

Start exploring the collection - download the first chapter of every title for free. 


\title{
Investigation of a plasma-target interaction through electric field characterization examining surface and volume charge contributions: modeling and experiment
}

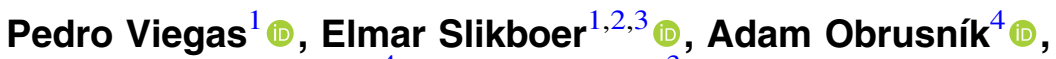 \\ Zdenek Bonaventura $^{4}\left({ }^{\circ}\right.$, Ana Sobota ${ }^{3}{ }^{\oplus}$, Enric Garcia-Caurel ${ }^{2}$, \\ Olivier Guaitella $^{1}$ (ib) and Anne Bourdon ${ }^{1,5}$
}

${ }^{1}$ LPP, CNRS, École Polytechnique, Sorbonne Université, Université Paris-Sud, L'Observatoire de Paris, Université Paris-Saclay, PSL Research University, F-91128 Palaiseau, France

${ }^{2}$ LPICM, CNRS, École Polytechnique, Université Paris-Saclay, F-91128 Palaiseau, France

${ }^{3}$ Department of Applied Physics, EPG, Eindhoven University of Technology, The Netherlands

${ }^{4}$ Faculty of Science, Masaryk University, Brno, Czechia

E-mail: pedro.viegas@1pp.polytechnique.fr, elmar.slikboer@lpp.polytechnique.fr, olivier.guaitella@lpp. polytechnique.fr and anne.bourdon@lpp.polytechnique.fr

Received 12 April 2018, revised 1 August 2018

Accepted for publication 24 August 2018

Published 14 September 2018

\begin{abstract}
Numerical simulations and experiments are performed to better understand the interaction between a pulsed helium plasma jet and a dielectric target. The focus of this work lies on the volume and surface charge influence on the electric field distribution. Experimentally, the electric field due to surface charges is measured inside an electro-optic target under exposure of a plasma jet, using the optical technique called Mueller polarimetry. For the first time, the timeresolved spatial distributions of both the axial and radial components of electric field inside the target are obtained simultaneously. A 2D fluid model is used in a complementary way to the experiments in order to study separately the contribution of volume charges and surface charges to the spatio-temporal evolutions of the electric field during the plasma-surface interaction. The experimental investigation shows that the average axial and radial components of electric field inside the dielectric target, only due to surface charges, are lower than generally reported for electric field values in the plasma plume. Thanks to the phenomenological comparison with experiments, simulations show that during the plasma-surface interaction two effects sequentially determine the electric field inside the target: firstly, a relatively high electric field is observed due to the proximity of the ionization front; afterwards, in longer timescales, lower electric fields are induced due to the contribution of both leftover volume charges close to the target and surface charges deposited on its surface. The experimental technique provides a unique way to examine this second phase of the plasma-surface interaction.
\end{abstract}

Keywords: plasma target interaction, plasma dielectric interaction, plasma jet, electric field, surface charges, Mueller polarimetry, electro-optic crystals

5 The author to whom any correspondence should be addressed

\section{Introduction}

The investigation of interactions between non-thermal plasmas at atmospheric pressure and surfaces is of great interest due to an increasing number of applications for these 
discharges. These applications include biomedical treatment (Fridman et al 2008, Kong et al 2009, Fridman and Friedman 2013, Tanaka and Hori 2017, Weltmann and von Woedtke 2017), surface functionalization (Noeske et al 2004, Cheng et al 2006, Fanelli and Fracassi 2017), catalytical interaction (Guaitella et al 2006, Neyts 2016), microbial sterilization (Seo et al 2010, Ehlbeck et al 2011), actuators for flow control (Orlov et al 2008, Unfer and Boeuf 2009) and nitrification of liquids and treatment for agriculture (Lindsay et al 2014). Most of the targets in these applications have a dielectric character. The plasmas used for these applications are often generated using a plasma jet, plane-to-plane dielectric barrier discharge (DBD) or surface DBD. Although homogeneous plasmas have been generated at atmospheric pressure with DBDs, most discharges generated and applied to surfaces for the given examples are filamentary and transient.

The variety of applications results directly from the complexity of the plasma-surface interactions (Laroussi and Leipold 2004, Neyts 2016). Firstly, radical species are produced in gas phase and transported to the surface (Graves 2012, Tian and Kushner 2014, Lindsay et al 2015). Additionally, materials are heated by the plasma interaction and UV radiation is provided by the discharge (Weltmann et al 2009). Finally, electric fields are generated inside the target and charge is deposited on its surface (Stollenwerk et al 2007, Sobota et al 2013).

In the past, several studies have taken place on filamentary discharges interacting with dielectric materials. In Allen and Mikropoulos (1999), Sobota et al (2009), Goldberg et al (2015) the interaction of streamer discharges with dielectric surfaces parallel to discharge propagation has been investigated experimentally and it has been shown that discharges propagate over the surface rather than through the gas. When dielectrics are placed as perpendicular targets instead, the discharge always propagates first through the gas before the interaction starts with the dielectric. Sakiyama et al (2008) have numerically investigated a helium plasma needle interacting with different surfaces and their results suggest that the plasma structure strongly depends on the electrical properties of the treated surface. Likewise, in Guaitella and Sobota (2015) it has been shown experimentally that the electrical characteristics of a dielectric target strongly modify the interaction with a plasma jet and the influence of the local enhancement of electric field. In Celestin et al (2009), filamentary air discharges impacting on a dielectric surface have been studied both experimentally and numerically and the role of volume and surface charges on the discharge structure has been discussed. Then, in Pechereau et al (2012), Pechereau and Bourdon (2014), a discharge in air in point-toplane configuration has been numerically investigated and the influence of the capacitance of the dielectric layer on the velocity of the discharge propagation on the surface has been studied. Moreover, the radial spreading of the discharge over the dielectric surface has been described in detail for both polarities of applied voltage. Considering the particular case of helium plasma jets, Norberg et al (2015) have studied numerically the impact on dielectric and metal surfaces with a voltage pulse of negative polarity. The approach of the ionization waves has been described, followed by the spreading on the target surface in the case of dielectric targets of different permittivities. Furthermore, the resulting electric field distribution inside the target has been reported. Other studies have described numerically the propagation of a helium jet and its interaction with a dielectric target, such as Breden and Raja (2014), Wang et al (2016), Ning et al (2018). Concerning plasma-surface interaction, these works have mostly investigated the influence of several physical parameters on the global discharge dynamics and on the fluxes of reactive species to the surface.

Other experimental studies have focused on the examination of surface charges on dielectric surfaces exposed to discharge impact. Surface charges can be investigated with Mueller polarimetry using electro-optic crystals, since their refractive index changes linearly with the induced electric field. The measured change in refractive index can be used to visualize the electric field to which the crystals are exposed to. As the electric field values are solely due to charge deposition on the surface, an estimation of the surface charge densities can be made when a homogeneous field approximation is applicable or a homogeneously charged disk is considered (Slikboer et al 2017). The visualization of electric field or surface charge density using electro-optic targets has been applied by Kawasaki et al (1991), Zhu et al (1995) to study the propagation of streamers on dielectrics. It has also been used with plasma jets by Sobota et al (2013), Wild et al (2014) and DBDs by Stollenwerk et al (2007), Bogaczyk et al (2012), Tschiersch et al (2014). The conventional investigation of the electro-optic crystals allows for the detection of the electric field perpendicular to the surface, following the Pockels effect that relates the refractive index of the crystal to the induced electric field. Recent developments show the possibility to simultaneously measure and decouple all electric field components (axial and radial) inside the material. This is done by examining the crystals not at normal incidence but at a $45^{\circ}$ angle, see Slikboer et al (2018b). Additionally, temperature profiles are detectable due to changes in the refractive index following the photo-elastic effect caused by the internal stress induced by temperature gradients, see Slikboer et al (2018a). The simultaneous detection of the axial and radial components of the electric field is an important tool to investigate the dynamics of the discharge propagation on the surface. Still, the cause of the surface charging itself is not well understood. Visualization of the electric field does not give information about the charged species that are deposited, nor about volume charge densities in the vicinity of the surface. Additionally, processes that cause charge removal at the surface are merely hypothesized. Lastly, it is unknown how the measured field, which is averaged throughout the thickness of the crystals, relates to the surface electric field value and the field in the plasma plume. This is important because filamentary and transient discharges are applied, and as such the homogeneous field approximation is probably not applicable.

To better understand the total dynamics of the plasmasurface interaction, both surface charges and volume charges 
in proximity of the target have to be known. Volume charges and electric field influence each other, induce surface charges and determine the discharge dynamics to a great extent. However, the measurement of volume charges in these filamentary transient discharges is difficult. In Bourdon et al (2016), Viegas et al (2018), it has been shown the interest to use plasma jet experiments and simulations in a complementary way to derive electric field values in locations where it is difficult to carry out experiments. In this work, the influence of volume and surface charges is investigated numerically, following the electric field associated to each type of charge. The calculation of separate contributions to the potential and electric field distributions is an approach that has been used in other works, such as Kulikovsky (1997), Celestin et al (2009), Pechereau and Bourdon (2014). This electric field characterization is not only focused on surface and volume charge contributions, but also on the spatial and temporal distributions of the axial and radial components of electric field in the plasma plume and inside the target. This study is complemented by the experimental measurement of both axial and radial time-resolved electric field patterns inside an electro-optic target under exposure of a plasma jet using Mueller polarimetry.

Both the numerical and experimental studies investigate the plasma-surface interaction by using a pulsed atmospheric pressure plasma jet propagating towards a dielectric target at $10 \mathrm{~mm}$ distance from the end of the tube. The focus lies on the interaction of the plasma plume with the dielectric target, and not on the propagation inside the tube. In section 2 , we present the experimental setup assessed in this paper, show basic imaging of the plasma plume and plasma-surface interaction and describe the method for electric field measurement inside the target. Then, in section 3, we describe the 2D fluid model used in this work. The electrode configuration, pulse duration and dielectric target permittivity are not exactly the same in simulations and experiments, and then this work is not a quantitative comparison between experiments and simulations. We have performed with the model a study of the impact of different geometries, flows, target permittivities and electron emission conditions and we have verified that the discharge propagation in the plasma plume and the impact on the surface of the target are not always centered on the axis of symmetry. Likewise, not all the conditions allow to describe the radial spreading of the discharge on the target. Thus, we have chosen the conditions where a centered discharge front impacts a dielectric surface and spreads on it, keeping the same timescales of discharge propagation as in the experiments. Then, in this work, we carry out a phenomenological study with both experiments and simulations to better understand the role of the averaging over the dielectric thickness in the experiments and the importance of the electric field induced by surface charges in comparison to the total electric field in the dielectric target. In section 4.1, the electric field measurements inside the target during the $1 \mu$ s pulse of applied voltage and some hundreds ns after the pulse are evaluated. Then, in section 4.2 we discuss simulation results on the interaction between a plasma plume and a dielectric target on the same timescale as in experiments. In particular, the dynamics between the charges and electric fields in the plasma plume and inside the target is assessed. Finally, in section 5, the experimental and numerical results are used in a complementary way to provide further detail on the influence of the averaging over the dielectric thickness in experiments and of volume and surface charges on the electric field distribution.

\section{Experimental investigation of plasma-target interaction}

In this work, the plasma-target interaction is examined experimentally by monitoring the propagation of a non-thermal atmospheric pressure plasma jet towards a dielectric glass target. Additionally, an electro-optic crystal is used to visualize the electric field inside the target under exposure of the plasma.

The jet design used in this experiment is described more comprehensively in Sobota et al (2013). The plasma jet operates in coaxial configuration where $1 \mathrm{slm}$ (standard liter per minute) helium flows first through an inner stainless steel tube into the pyrex capillary (inner diameter $2.5 \mathrm{~mm}$ and outer $4.0 \mathrm{~mm}$ ) before it mixes with air. The inner tube operates as powered electrode driven by a $1 \mu$ s pulse of $4 \mathrm{kV}$ at a $5 \mathrm{kHz}$ repetition rate with a rise-time of $50 \mathrm{~ns}$. The ionization wave travels from the edge of the inner tube towards the grounded ring, which is $5 \mathrm{~mm}$ downstream around the pyrex capillary. After charging the dielectric tube between the powered and grounded electrodes, the ionization wave travels further downstream, creating a plasma plume appearing outside the tube. The discharge is forced to interact with the dielectric target perpendicular to its propagation. This is visualized by capturing the light emission from the plasma as the ionization wave travels towards the $0.5 \mathrm{~mm}$ thick glass target and spreads over its surface, as seen in figure 1 for different time delays $t_{d}$ relative to the rise of the voltage pulse, using $25 \mathrm{~ns}$ exposure frames.

The gap between the end of the capillary and the glass target is $10 \mathrm{~mm}$. When the front of the ionization wave reaches the target, it starts propagating radially on the surface. The glass target is not grounded and as such is at floating potential. At the surface, the radial spreading corresponds firstly to a disk of increasing diameter. At $625 \mathrm{~ns}$, when the disk already has more than $2 \mathrm{~mm}$ of diameter, the spreading splits into several streamer branches, reproducible in number for a given voltage and gas flow. This is confirmed with topview imaging, complementary to the side-view imaging shown in figure 1 . At the same time, light emissions start to diminish. To evaluate the charging at the surface of the target, Mueller polarimetry is applied to examine an electro-optic target $\left(\mathrm{Bi}_{12} \mathrm{SiO}_{20}\right)$ under exposure of an ionization wave generated by the same plasma jet. The refractive index of electro-optic crystals changes linearly with the applied electric field induced by surface charges, according to the Pockels effect. The dielectric constant of the crystal used for this electric field measurement is 56, and therefore higher than that of the glass target used for the imaging $\left(\epsilon_{r} \sim 4\right)$. With 


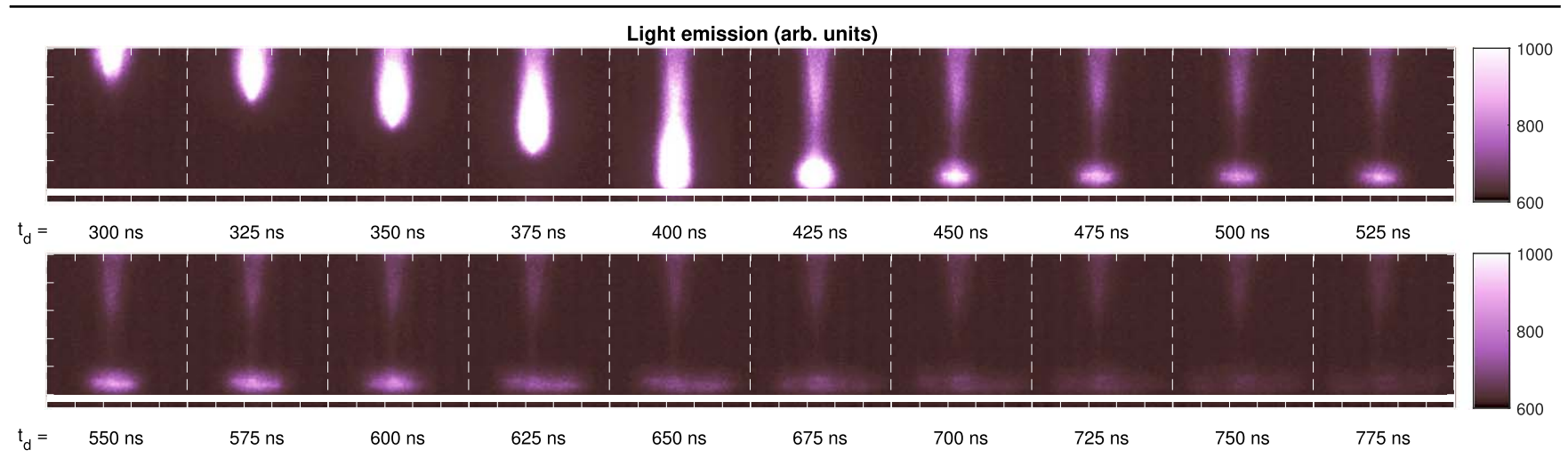

Figure 1. Imaging of light emission showing the discharge propagation between the end of the capillary tube (on top) and the target (bottom white layer), visualizing the impact on the target surface, at several time delays $t_{d}$ relative to the rise of the voltage pulse.

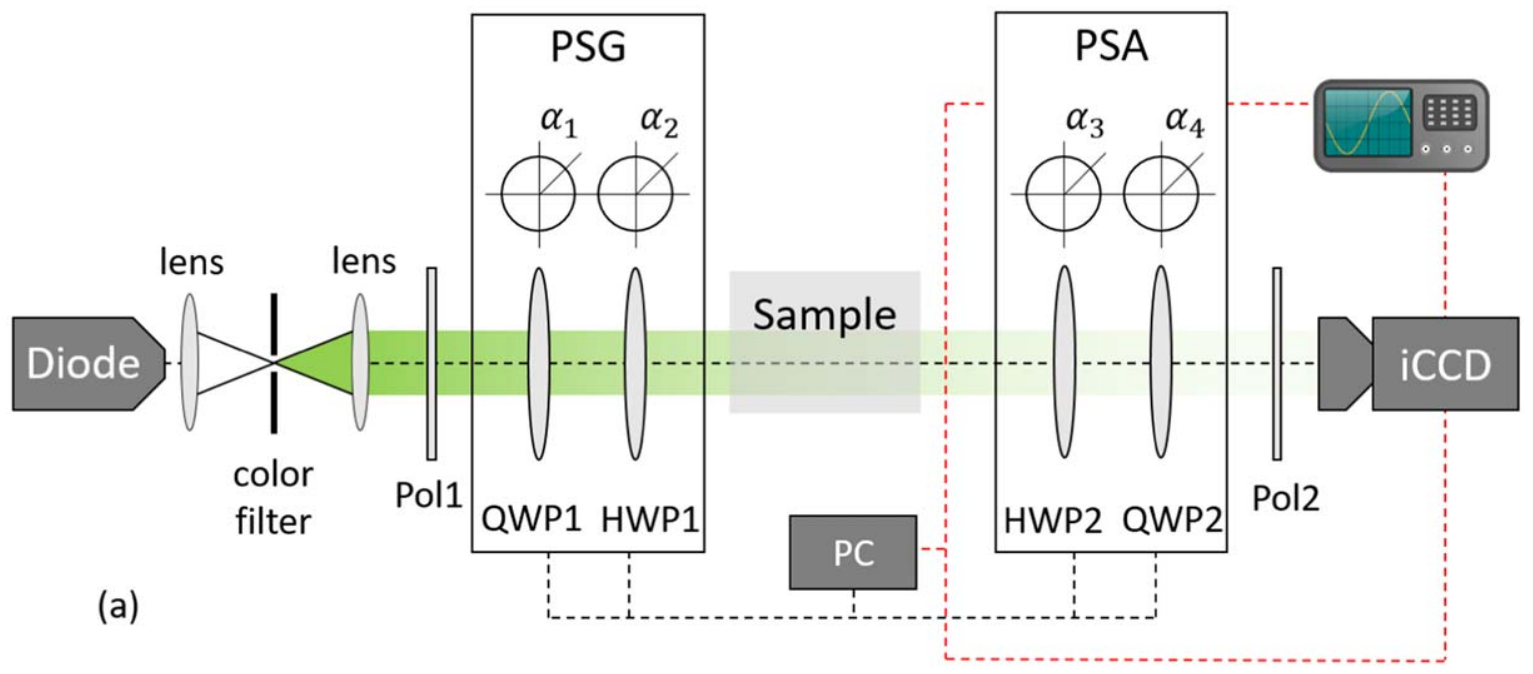

(a)

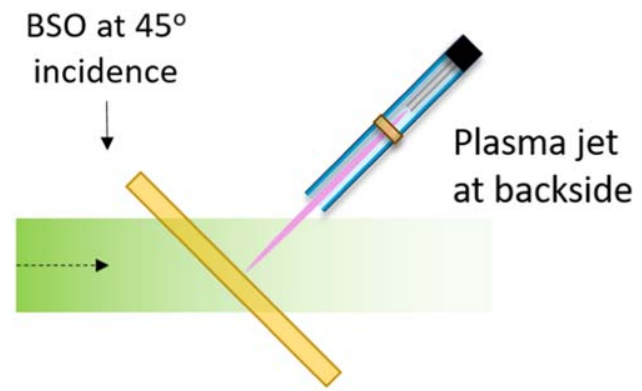

(c)

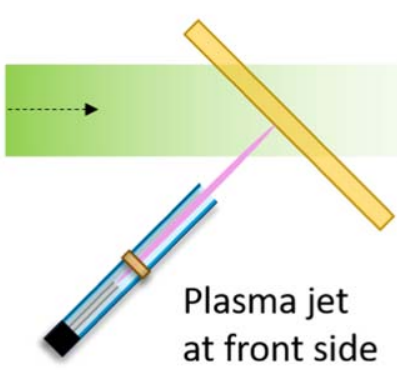

Figure 2. The experimental setup used for the electric field measurements inside an electro-optic $\mathrm{Bi}_{12} \mathrm{SiO}_{20}$ crystal. The Mueller polarimeter, figure 2(a), investigates the Mueller matrix of a sample using two liquid crystals in both the polarizer state generator (PSG) and the polarizer state analyzer (PSA). Figures 2(b) and (c) show two different approaches used to investigate the BSO crystal at a $45^{\circ}$ angle, impacted by the plasma jet.

Mueller polarimetry it is possible to obtain images of the induced electric fields inside the target, following the measurement of the Mueller matrix of the target (Slikboer et al 2018a). Polarized light is used to characterize the optical properties of the material captured within the Mueller matrix in terms of diattenuation, depolarization and birefringence (Garcia-Caurel et al 2013). The latter depends on the induced change of refractive index, making it possible to retrieve the electric field. The imaging Mueller polarimeter used in this work is more comprehensively discussed in Slikboer et al (2018a, 2018b).
As shown in figure 2, the experimental setup consists of a polarizer state analyzer (PSA) and polarizer state generator (PSG). Both consist of two ferro-electric liquid crystals that can be externally operated to control their orientations. Their phase retardance relates to that of a quarter and half waveplate. The orientation of their optical axis can be modified during operation of the instrument and switched between two well-definite positions. Since we use four liquid crystals the sequential switching between all possible combinations allows for the acquisition of 16 images at the iCCD capturing the total transmission of the system. 
These images form the $4 \times 4$ intensity (imaging) matrix which can be transformed into the Mueller matrix of the sample placed in between the PSA and the PSG. This is done using the Mueller matrix of the PSA and PSG, which is obtained following the eigenvalue calibration method (Compain et al 1999, Macías-Romero and Török 2012). The Mueller matrix of the sample is analyzed to retrieve the birefringence induced by the ionization wave using the logarithmic decomposition (Azzam 1978, Ossikovski 2011). The total birefringence, also referred to as total retardance, is divided in the circular component $\Gamma_{c}$, the linear component in the $0 / 90$ coordinate system $\Gamma_{0 / 90}$ and the second linear component in the diagonal system $\Gamma_{45 / 135}$. A broader description of the meaning of these different birefringences can be found in literature, e.g. Garcia-Caurel et al (2013), Gil and Ossikovski (2016).

The advantage of Mueller polarimetry is exploited since the linear retardance is obtained along two different axis, i.e. $\Gamma_{0 / 90}$ and $\Gamma_{45 / 135}$. Each depends on the electric field in a different way. The birefringence can be examined with different experimental setups, e.g. a Sénarmont setup, but this only allows for the acquisition of one of the linear retardances instead of both. Analytically, the dependence between the linear retardances and the external electric field is obtained in Slikboer et al (2018b). This relation depends on the orientation of the crystal with regard to the incident light. Conventionally, $\mathrm{Bi}_{12} \mathrm{SiO}_{20}$ crystals are examined at normal incidence, which allows for the investigation of the axial electric field, parallel to the light propagation, $E_{z}$. Derived in Slikboer et al (2018b) is the different case where light propagates at a $45^{\circ}$ angle, as shown in figure 2(b). In this case, the two linear retardances depend on all three electric field components as:

$$
\begin{gathered}
\Gamma_{0 / 90}=\frac{2 \pi d^{*}}{\lambda} \frac{1}{2} n_{o}^{3} r_{41} E_{y}, \\
\Gamma_{45 / 135}=\frac{2 \pi d^{*}}{\lambda} \frac{1}{\sqrt{2}} n_{o}^{3} r_{41}\left(E_{z}-E_{x}\right) .
\end{gathered}
$$

The given relation depends on the distance light travels through the crystal $d^{*}=\sqrt{2} d=0.5 \sqrt{2} \mathrm{~mm}$, the index of refraction $n_{0}=2.54$, the wavelength of the light $\lambda=530 \mathrm{~nm}$, the electro-optic constant $r_{41}=4.8 \mathrm{pm} \mathrm{V}^{-1}$ and the individual electric field components. In this case, the $z$-axis is chosen normal to the surface of the material and as such not parallel to the propagation of the light, but parallel to the axis of the plasma jet. Since the retardance in the diagonal system scales with a combination of the radial $E_{x}$ and axial $E_{z}$, an additional measurement has to be done. $\Gamma_{0 / 90}$ and $\Gamma_{45 / 135}$ are measured with the plasma jet impacting on the front side of the material, i.e. the side facing the light source. Then, an additional measurement is done with the plasma jet on the back side of the material, facing the iCCD camera, as shown in figures 2 (b) and (c). Since only $E_{z}$ changes sign, all the electric field components can be separated from the measured retardances by simple addition and subtraction. Then, by varying the delay time of acquisition relative to the high voltage pulse, a time resolved investigation of the electric

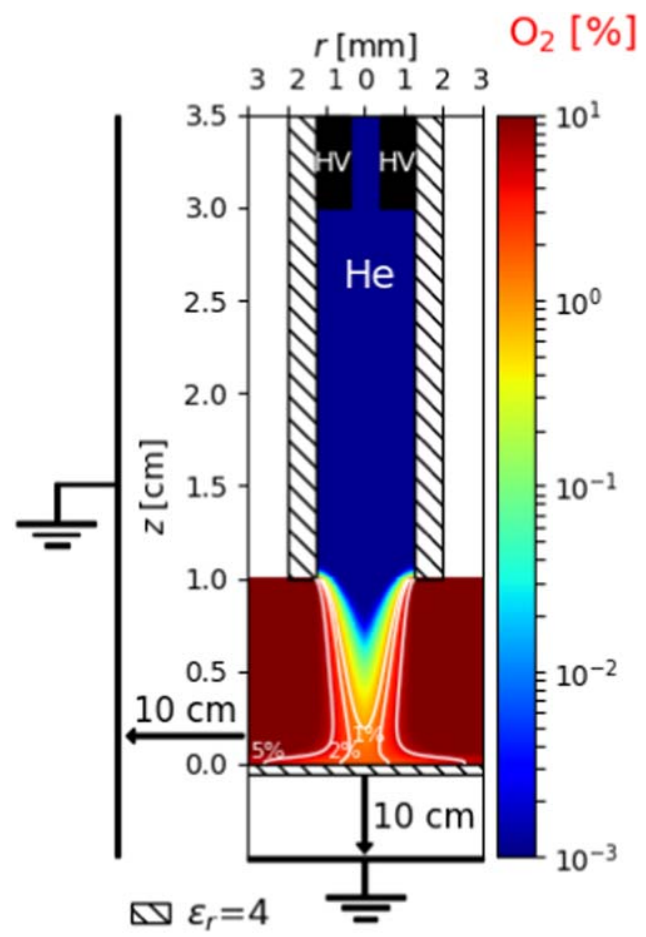

Figure 3. Side view schematics of the discharge set-up used in the simulations. The color plot and the white curves show the $\mathrm{O}_{2}$ spatial distribution in the $\mathrm{He}-\mathrm{O}_{2}$ mixture (percentage over a total of $2.45 \times 10^{19} \mathrm{~cm}^{-3}$ gas density).

field components inside the target is achieved. This optical technique gives a top view image of the surface charge induced electric field inside the target under exposure of the plasma jet, which is complementary to the side view imaging shown in figure 1. Results using this procedure will be shown in section 4 together with the numerical results of electric field characterization of the plasma-surface interaction in similar conditions.

\section{Model description and discharge dynamics of propagation}

\subsection{Model description}

The model set-up is shown in figure 3. As in the experiments, a dielectric quartz tube with relative permittivity of $\epsilon_{r}=4$, length $2.5 \mathrm{~cm}$ (between $z=1.0 \mathrm{~cm}$ and $z=3.5 \mathrm{~cm}$ ), internal radius $r_{\text {in }}=1.25 \mathrm{~mm}$ and outer radius $r_{\text {out }}=2.0 \mathrm{~mm}$ is used. Helium flows through the tube with 1 slm flux as in experimental conditions. Also, a dielectric glass target with $\epsilon_{r}=4$ is placed perpendicularly to the tube at $1 \mathrm{~cm}$ from the end of the tube, as in figure 1 . The target is defined as a cylinder of $2 \mathrm{~cm}$ radius and $0.5 \mathrm{~mm}$ thickness, set between $z=-0.5 \mathrm{~mm}$ and $z=0$. It is at a floating potential as in experiments, and a grounded plane is set $10 \mathrm{~cm}$ behind it. A ring electrode of inner radius $0.4 \mathrm{~mm}$ and outer radius $1.25 \mathrm{~mm}$ is set inside the tube between $z=3.0 \mathrm{~cm}$ and $z=3.5 \mathrm{~cm}$ and no grounded ring is wrapped around the glass tube. This electrode is powered by a positive pulsed voltage that rises in $50 \mathrm{~ns}$ to 
$4 \mathrm{kV}$, and then remains constant as in experiments. The pulse duration is chosen to be shorter than in experiments to avoid the streamer branching reported in section 2, that cannot be described with an axisymmetric model. Thus, the voltage remains constant until $t=800 \mathrm{~ns}$ and then decreases to 0 until $850 \mathrm{~ns}$. During the voltage rise and decrease, a linear variation of the voltage has been assumed. As the electrode geometry is slightly different from the experiments, the velocity of propagation is a bit lower in the simulations and consequently the time of impact on the target is higher. This difference does not matter for the purpose of this paper of studying the dynamics between charges and electric fields close to the target, since the structure of the plasma-target interaction is the same in simulations and experiments, as will be shown in section 3.2. Finally, figure 3 also shows that the discharge set-up is placed inside a grounded cylinder with a radius of $10 \mathrm{~cm}$. Between the dielectric target and the grounded plane, as well as between the dielectric tube and the grounded cylinder, the space is considered as a dielectric of air permittivity $\epsilon_{r}=1$. On the last boundary of the domain (i.e. at $z=3.5 \mathrm{~cm}$ ), the axial gradient of electric potential is set to zero. The calculation lasts until $t=2000 \mathrm{~ns}$, more than $1 \mu \mathrm{s}$ after the end of the pulse.

The computational domain is cylindrically symmetrical and the simulations have been carried out at atmospheric pressure and at $T=300 \mathrm{~K}$. To simulate the discharge dynamics we use as in Jánský and Bourdon (2014), Bourdon et al (2016), Viegas et al (2018), a 2D axisymmetric fluid model based on drift-diffusion-reaction equations for electrons, positive ions and negative ions and reaction equations for neutral species, coupled with Poisson's equation in cylindrical coordinates $(z, r)$ :

$$
\begin{gathered}
\frac{\partial n_{i}}{\partial t}+\nabla . \mathbf{j}_{\mathbf{i}}=S_{i}, \\
\mathbf{j}_{\mathbf{i}}=\left(q_{i} /|e|\right) n_{i} \mu_{i} \mathbf{E}-D_{i} \nabla n_{i}, \\
\epsilon_{0} \nabla \cdot\left(\epsilon_{r} \nabla V\right)=-\rho-\sigma \delta_{s}, \\
\mathbf{E}=-\nabla V ; \rho=\sum q_{i} n_{i},
\end{gathered}
$$

where the subscript $i$ refers to each species and $n_{i}, q_{i}, j_{i}, \mu_{i}$ and $D_{i}$ are the number density, the charge, the flux, the mobility and the diffusion coefficient of each species $i$, respectively. $S_{i}$ is the total rate of production and destruction of species $i$ by kinetic processes and by photoionization. $V$ is the potential, $\mathbf{E}$ the electric field, $e$ the electron charge, $\epsilon_{0}$ the vacuum permittivity, $\epsilon_{r}$ the relative permittivity and $\delta_{s}$ the Kronecker delta (equal to 1 on the dielectric/gas interfaces: tube and target). It is important to point out that the numerical resolution of Poisson's equation (5) includes not only the geometrical Laplacian potential distribution, defined by boundary conditions, but also the volume charge density $\rho$ and the surface charge density $\sigma$ contributions. At the surface of both the tube and the target, secondary emission of electrons by ion bombardment ( $\gamma=0.1$ for all ions) is taken into account. This relatively high value for this coefficient is a way to roughly take into account other secondary emission processes such as photoemission and secondary emission of electrons by impact of metastable species. Surface charge density $\sigma$ on the surface of the dielectrics is obtained by time integrating charged particle fluxes through electric drift to the surface. These charges then remain immobile on the surface of the dielectrics.

To model the experimental conditions described in section 2 , in which helium flows at $1 \mathrm{slm}$ through the tube into air, the plasma model was coupled with flow calculations. As a first approximation, the model considers a flow of $1 \mathrm{slm}$ of helium with $10 \mathrm{ppm}$ of $\mathrm{O}_{2}$ impurities flowing downstream into an $\mathrm{O}_{2}$ environment. Both experiments and simulations (Schmidt-Bleker et al 2015, Winter et al 2015) have shown that the use of $\mathrm{O}_{2}$ or air as surrounding gas for a $\mathrm{He}$ jet present similarities with respect to discharge dynamics in the plasma plume. In Naidis (2011), Schmidt-Bleker et al (2015) the important role of the electronegativity of the surrounding gas, either $\mathrm{O}_{2}$ or air, has been highlighted. The flow calculation solves the compressible Navier-Stokes equations with the buoyant force term, coupled to a diffusion equation describing the mixing of the gas components $\mathrm{He}$ and $\mathrm{O}_{2}$ and has been performed using the COMSOL software (COMSOL 2017). This flow model has been described in Arjunan et al (2016), Vorác et al (2014) and has been used previously in Sobota et al (2016). In the current conditions, the gas flow velocity is slightly higher than $3 \mu \mathrm{m} \mu \mathrm{s}^{-1}$. Therefore, the model takes a static gas hypothesis, relevant in the microsecond time-scale studied in this work, during which the gas flow can be considered to be frozen with no diffusion of neutral species. This approach of coupling a static-solution gas flow with a transient plasma model has been used in other works, such as Breden et al (2012), Naidis (2012). The spatial distribution of $\mathrm{O}_{2}$ in the $\mathrm{He}-\mathrm{O}_{2}$ mixture at atmospheric pressure and at $T=300 \quad \mathrm{~K} \quad$ (total gas density $N=2.45 \times 10^{19} \mathrm{~cm}^{-3}$ ) obtained from the flow calculation is presented in figure 3 . There are also added the contour curves of 1,2 and $5 \% \mathrm{O}_{2}$ in the $\mathrm{He}-\mathrm{O}_{2}$ mixture, to point out the mixture region studied in this work.

The reaction scheme proposed in Liu et al (2010) is used to describe the kinetics of electrons, helium and oxygen positive ions, oxygen negative ions and neutral species in the $\mathrm{He}-\mathrm{O}_{2}$ plasma, as in Viegas et al (2018). The scheme includes a total of 288 reactions with 5 positive ions, 5 negative species and 11 neutral species: e, $\mathrm{He}^{+}, \mathrm{He}_{2}^{+}, \mathrm{O}^{+}, \mathrm{O}_{2}^{+}$, $\mathrm{O}_{4}^{+}, \mathrm{O}^{-}, \mathrm{O}_{2}^{-}, \mathrm{O}_{3}^{-}, \mathrm{O}_{4}^{-}, \mathrm{He}, \mathrm{He}\left(2^{3} \mathrm{~S}, 2^{1} \mathrm{~S}\right), \mathrm{He}_{2}{ }^{*}, \mathrm{O}, \mathrm{O}\left({ }^{1} \mathrm{D}\right)$, $\mathrm{O}\left({ }^{1} \mathrm{~S}\right), \mathrm{O}_{2}, \mathrm{O}_{2}\left(\mathrm{a}_{1} \Delta_{g}\right), \mathrm{O}_{2}\left(\mathrm{~b}^{1} \Sigma_{g}^{+}\right), \mathrm{O}_{2}(v=1-4), \mathrm{O}_{3}$. The electron swarm parameters and the reaction rates of electron impact excitation and ionization reactions are functions of both the local gas mixture and the local reduced electric field $E / N$. These coefficients are taken for $13 \mathrm{He}-\mathrm{O}_{2}$ gas mixtures (percentage of $\mathrm{O}_{2}: 0.001 \%, 0.01 \%, 0.1 \%, 0.25 \%, 0.5 \%$, $0.75 \%, 1 \%, 2.5 \%, 5 \%, 7.5 \%, 10 \%, 50 \%, 100 \%$ ) and $210 \mathrm{E} / \mathrm{N}$ values between 0.1 and $1000 \mathrm{Td}$ from the electron Boltzmann equation solver BOLSIG+ (Hagelaar and Pitchford 2005), using the IST-Lisbon database of cross sections in LXCat (Pancheshnyi et al 2012, IST 2018), and tabulated. In each cell and at each timestep, the model finds the tabulated values of gas mixture and $E / N$ that are closer to the local values. Then, each coefficient $k$ is calculated for the local value of $E$ / $N$ and the closest tabulated values of gas mixture by linear 
interpolation between the upper and lower tabulated values of $E / N$ and we obtain $k_{\text {mixdown }}(E / N)$ and $k_{\text {mixup }}(E / N)$. Finally, each coefficient is linearly interpolated between these two for the local value of gas mixture and we obtain $k(\operatorname{mix}, E / N)$. Positive and negative ion mobilities are retrieved from LXCat databases (Ellis et al 1976, Pancheshnyi et al 2012) as function of $E / N$ as in Viegas et al (2018). Diffusion coefficients for ions are evaluated using the Einstein relation. For the photoionization model, we use the approach described in Viegas et al (2018) with $A_{\mathrm{ph}}=10 \times X_{\mathrm{O}_{2}}$. As repetitive voltage pulses are used in the previously described experiments $(f=5 \mathrm{kHz})$, we take into account a uniform initial preionization density $n_{\text {init }}=10^{9} \mathrm{~cm}^{-3}$ of electrons and $\mathrm{O}_{2}^{+}$ positive ions. However, no initial surface charges are considered on the dielectric surfaces, which will be shown in section 4 to be a reasonable assumption, due to the almost zero surface charge densities measured and calculated after the end of the pulse of applied voltage.

A finite volume approach and a cartesian mesh are used. The mesh size is $10 \mu \mathrm{m}$ axially between $z=-0.05 \mathrm{~cm}$ and $z=3.5 \mathrm{~cm}$ and radially between $r=0$ and $r=3.0 \mathrm{~mm}$. Then, both behind the target and for $r>3.0 \mathrm{~mm}$, the mesh size is expanded using a geometric progression until reaching the boundaries of the computational domain at $r=10 \mathrm{~cm}$ and $z=-10 \mathrm{~cm}$. This refinement requires a mesh of $n_{z} \times n_{r}=3600 \times 370=1.332$ million points. The average computational time required for a simulation run to obtain the results presented in this paper was of three days with 32 MPI processes on a multicore cluster 'Hopper'(32 nodes DELL C6200 bi-pro with two 8-core processors, 64 Go of memory and $2.6 \mathrm{GHz}$ frequency per node). The numerical solvers used in the code are the same as in Bourdon et al (2016). Further details on the numerical schemes and other characteristics of the simulations (boundary conditions and time-step calculation) are given in Pechereau (2013).

\subsection{Simulation results of discharge dynamics of propagation}

With the model described in section 3.1, the discharge ignites close to the powered electrode at $z=3.0 \mathrm{~cm}$ and $t=50 \mathrm{~ns}$ and then propagates until the end of the tube at $z=1.0 \mathrm{~cm}$ and $t=425 \mathrm{~ns}$. The discharge reaches the end of the tube with a centered structure, having the maximum of the axial component of electric field positioned on the axis of symmetry. Then, the discharge dynamics in the plasma plume, between the end of the tube and the dielectric target, is represented in figure 4. This figure shows the spatial distribution at several times of two quantities that are representative of discharge propagation: the magnitude of the electric field, calculated from its axial and radial components $E_{t}=\sqrt{E_{z}^{2}+E_{r}^{2}}$; and the source term of ionization through electron impact $S_{e}$, obtained from ionization reactions through collisions of electrons with all the neutral species listed in section 3.1. The spatial distribution of $S_{e}$ is comparable with the light emission during discharge propagation, presented in figure 1 . There is also presented the contour curve of $5 \% \mathrm{O}_{2}$ in the $\mathrm{He}-\mathrm{O}_{2}$ mixture, to clearly show the mixture region where the discharge dynamics takes place.
As the discharge front has a centered profile of electric field at the end of the tube, it continues propagation in the plasma plume with a centered structure. This feature is visible in figure 4 at $510 \mathrm{~ns}$ when the front is in the middle of the tube-target gap and at $t=625 \mathrm{~ns}$ as the discharge impacts the target, as well as in the experimentally obtained imaging in figure 1. In fact, as the discharge front crosses the tube-target gap, it is passing through several layers of $\mathrm{O}_{2}$ admixture in the $\mathrm{He}-\mathrm{O}_{2}$ mixture, from $10 \mathrm{ppm}$ at $z=10 \mathrm{~mm}$, up to $1.8 \%$ at $r=0$ on the target surface. The axial electric field on the discharge front increases during the propagation towards the surface, reaching up to $35 \mathrm{kV} \mathrm{cm}^{-1}$ on the target surface, as shown in figure 4. After the discharge impact on the target, the plasma spreads radially on the dielectric surface and the front reaches a radial position of approximately $1.8 \mathrm{~mm}$ at 800 ns when the applied voltage starts decreasing and the spreading significantly slows down.

\section{Electric field characterization in plasma-dielectric interaction}

\subsection{Experiment: electric field inside the dielectric target}

In this section, the results of electric field measurement inside the target using the method described in section 2 are presented.

As explained, Mueller polarimetry allows for the investigation of electric field by the analysis of the birefringent properties obtained by applying the logarithmic decomposition to the measured Mueller matrix. The resulting separate electric field components are shown in figure 5 for different time delays $t_{d}$ relative to the rise of the voltage pulse. For the first time, the spatial distributions of both the axial and radial components of electric field inside the target due to $\sigma$ are measured simultaneously. Exposure times of 100 ns are used and every intensity frame needed to construct the Mueller matrix consists of 200 acquisitions to reduce noise via averaging. The axial $E_{z}$ field is shown to be positive, resulting from positive charge deposition on the front side of the crystal. The axial component $E_{z}$ and the radial component $E_{x}$ are obtained from $\Gamma_{45 / 135}$ measured with the jet on front and back side. The other radial component $E_{y}$ is retrieved using the $\Gamma_{0 / 90}$. Both retardances are part of the Mueller matrix and as such measured simultaneously using Mueller polarimetry. The $z$-axis is parallel to the axis of propagation of the plasma jet, and perpendicular to the surface.

It is interesting to notice that the discharge propagation towards targets with different permitivities presents the same general dynamics. Figure 1 shows the ionization waves generated experimentally impact on the glass $\left(\epsilon_{r}=4\right)$ surface at $t_{d}=400 \mathrm{~ns}$. The electric field inside the electro-optic crystal, shown in figure 5, indicates the impact on the BSO $\left(\epsilon_{r}=56\right)$ surface at $t_{d}=400 \mathrm{~ns}$ as well. All electric fields shown in figure 5 are induced by surface charge density $\sigma$. A continuous charging of the surface is observed with a radial spreading following the ionization front from the moment of impact at $t_{d}=0.4 \mu \mathrm{s}$ until the end of the pulse at $t_{d}=1.0 \mu \mathrm{s}$. 

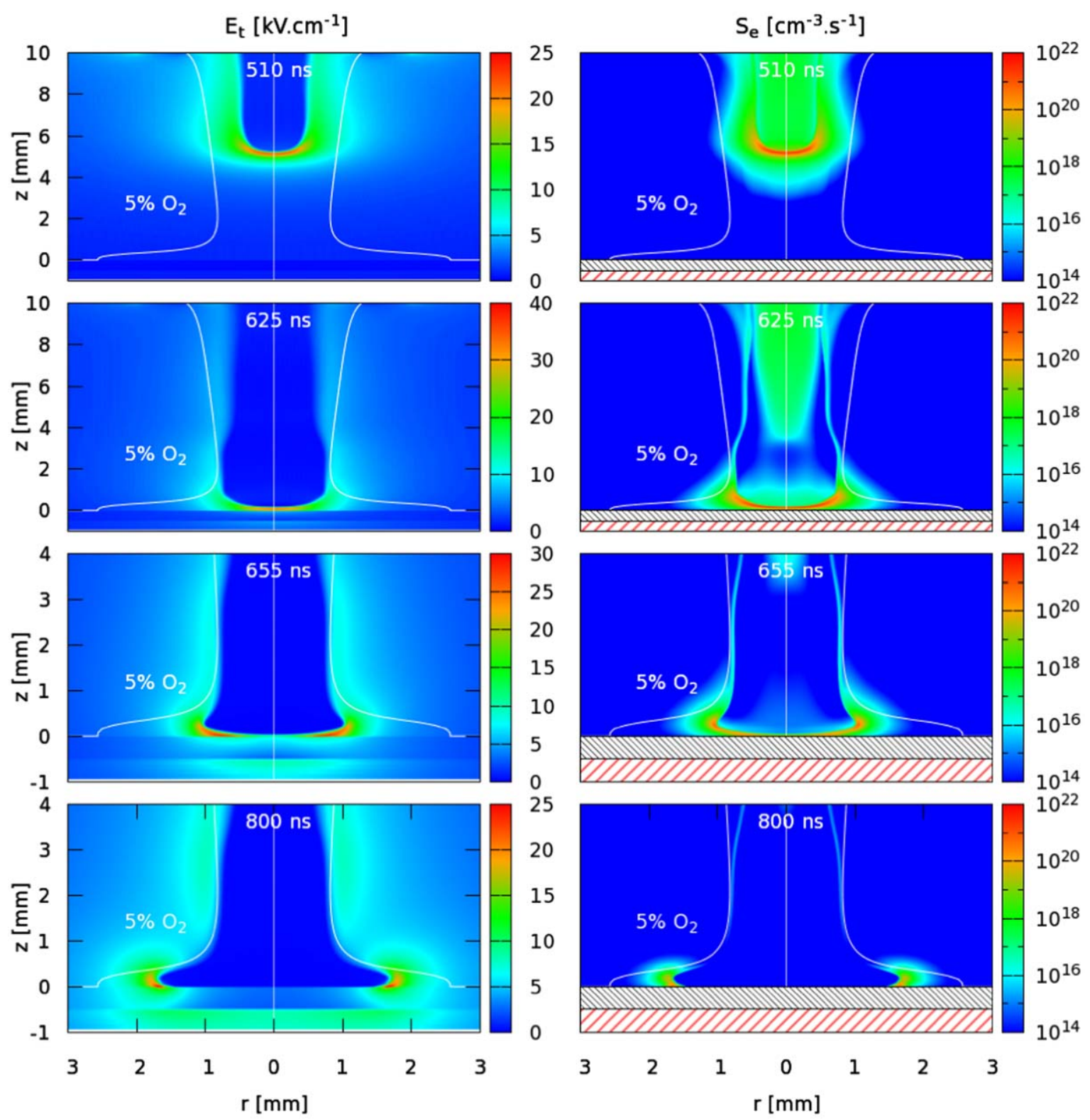

Figure 4. Cross sectional views representative of discharge propagation in the plasma plume and spreading on the target surface, at $t=510$, 625,655 and $800 \mathrm{~ns}$. On the left, the magnitude of the electric field $E_{t}$; and on the right, the value of the electron-impact ionization source term $S_{e}$. The figures are zoomed-in axially in time to see the approach to the target, indicated by a black stripped rectangle.

In the center of the impact region, constant fields, indicating stable surface charge densities, are observed for several hundreds ns until the pulse ends. Then, the electric field components diminish starting from the center of the impact region and it takes approximately $0.4 \mu \mathrm{s}$ for the surface charges to be removed to a great extent. Constant fields due to surface charges have been measured for longer timescales, up to $10 \mu \mathrm{s}$, with sinusoidal applied voltage of $30 \mathrm{kHz}$ frequency (Slikboer et al 2016). The observed maximum value of $E_{z}$ is $5 \mathrm{kV} \mathrm{cm}^{-1}$, measured in the center of the impact point. This value is higher than the radial component of $3 \mathrm{kV} \mathrm{cm}^{-1}$. A homogeneous spreading is observed within the first $300 \mathrm{~ns}$ after impact. This area where charge is deposited remains smaller than $2 \times 2 \mathrm{~mm}^{2}$. Afterwards, patterns from radial surface streamers are observed, enlarging the total area of the plasma-surface interaction. Charge deposited by the surface streamers are the last to be removed.

The values of electric field measured are an average throughout the optical path that the light has traveled. As such, the value of electric field on the target surface due to surface charges is expected to be higher. The ratio between the surface value and the measured value as shown in figure 5 depends on the size of the charged region and the thickness of the crystal (Slikboer et al 2017). The measured electric field experienced by the target due to surface charges is lower than the one in the plasma plume reported in Sobota et al (2016), of around $18 \mathrm{kV} \mathrm{cm}^{-1}$ (measured by Stark polarization spectroscopy in the plasma plume of a freely expanding jet with a lower applied voltage). To understand how these fields relate to each other, the model described in section 3.1 is used 

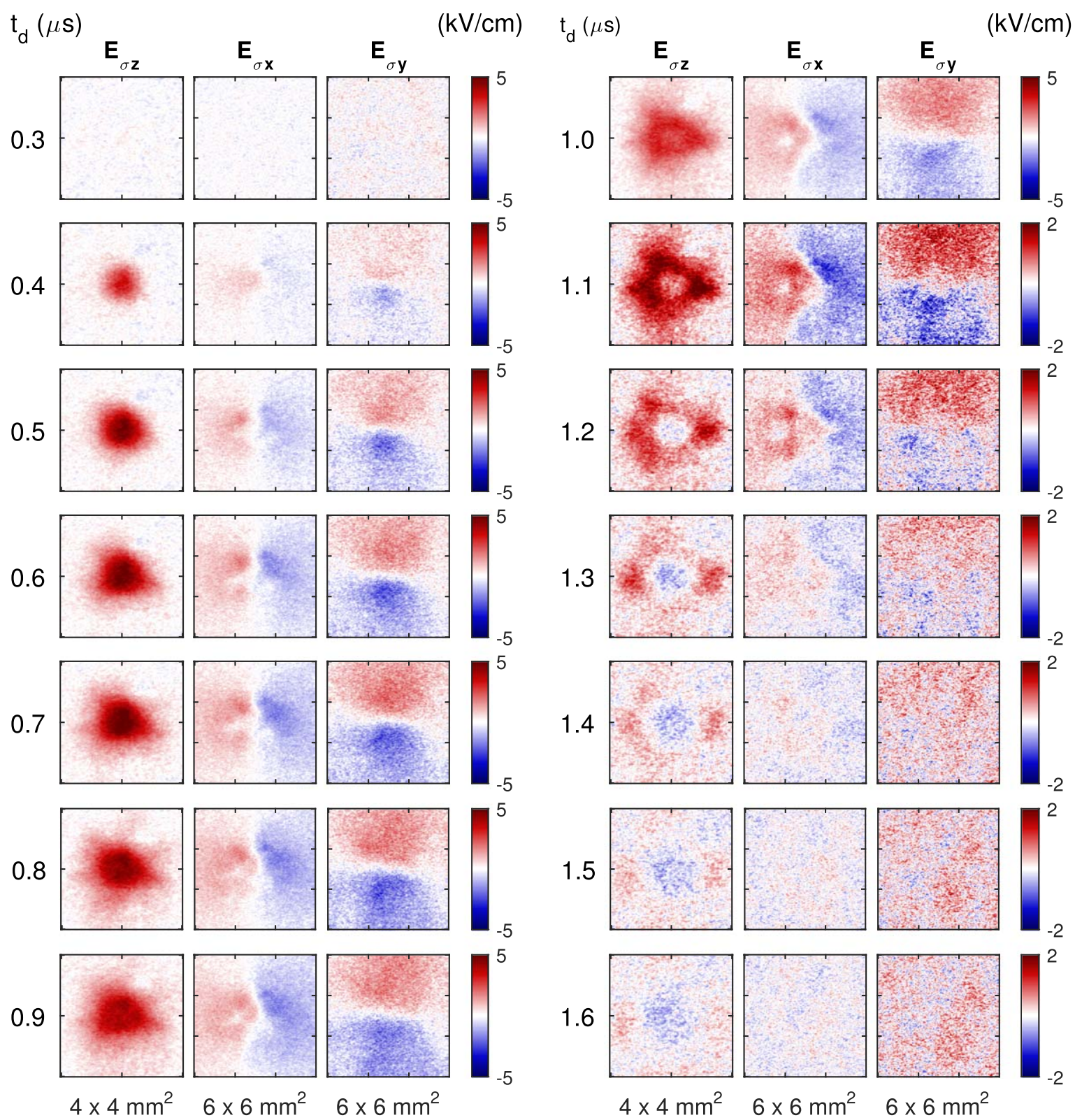

Figure 5. The obtained electric field patterns (axial $E_{z}$ and radial $E_{x}$ and $E_{y}$ ) inside the electro-optic target (top-view), due to surface charge $\sigma$ deposited by the ionization wave. The pulse ends at $t_{d}=1 \mu \mathrm{s}$. Afterwards, a different color scale is used. The spatial dimensions of each pattern are either $4 \times 4 \mathrm{~mm}^{2}$ or $6 \times 6 \mathrm{~mm}^{2}$, indicated with $2 \mathrm{~mm}$ spacing, with the center of the images at the same position.

for a phenomenological evaluation of the electric field distribution.

\subsection{Model: electric field distribution in plasma-dielectric interaction}

In this section, we use the model to separate the contributions of volume and surface charges to the electric field in the plasma plume and in the dielectric target. First, figure 6 shows the spatial distribution of the volume charge density $\rho$, defined in equation (6), and of the axial $E_{z}$ and radial $E_{r}$ electric field components at several moments, focused in the region between $0.4 \mathrm{~mm}$ above the target surface and $0.4 \mathrm{~mm}$ axially inside the target. The radial profiles of surface charge density $\sigma$ are represented for the same instants in time in figure 7 .

In figure 6 we first notice the discharge arrival on the target at $t=625 \mathrm{~ns}$. The positive volume charge density $\rho$ exhibited there is created by the charge separation on the discharge front and, on its turn, originates the electric field components present in the same figure for $t=625 \mathrm{~ns}$. A downwards-directed axial component $E_{z}$ is created between $\rho$ and the grounded plane at $z=-10 \mathrm{~cm}$ and therefore gives rise to a flux of positive charged species towards the target. In figure 7 it is shown that there is a centered charge deposition during $30 \mathrm{~ns}$ due to the mentioned $E_{z}$, increasing $\sigma$ from approximately zero at $t=625 \mathrm{~ns}$ to $\sim 1.5 \mathrm{nC} \mathrm{cm}^{-2}$ on the axis of symmetry at $t=655 \mathrm{~ns}$. We have checked that the positive species deposited are dominantly oxygen ions, which 

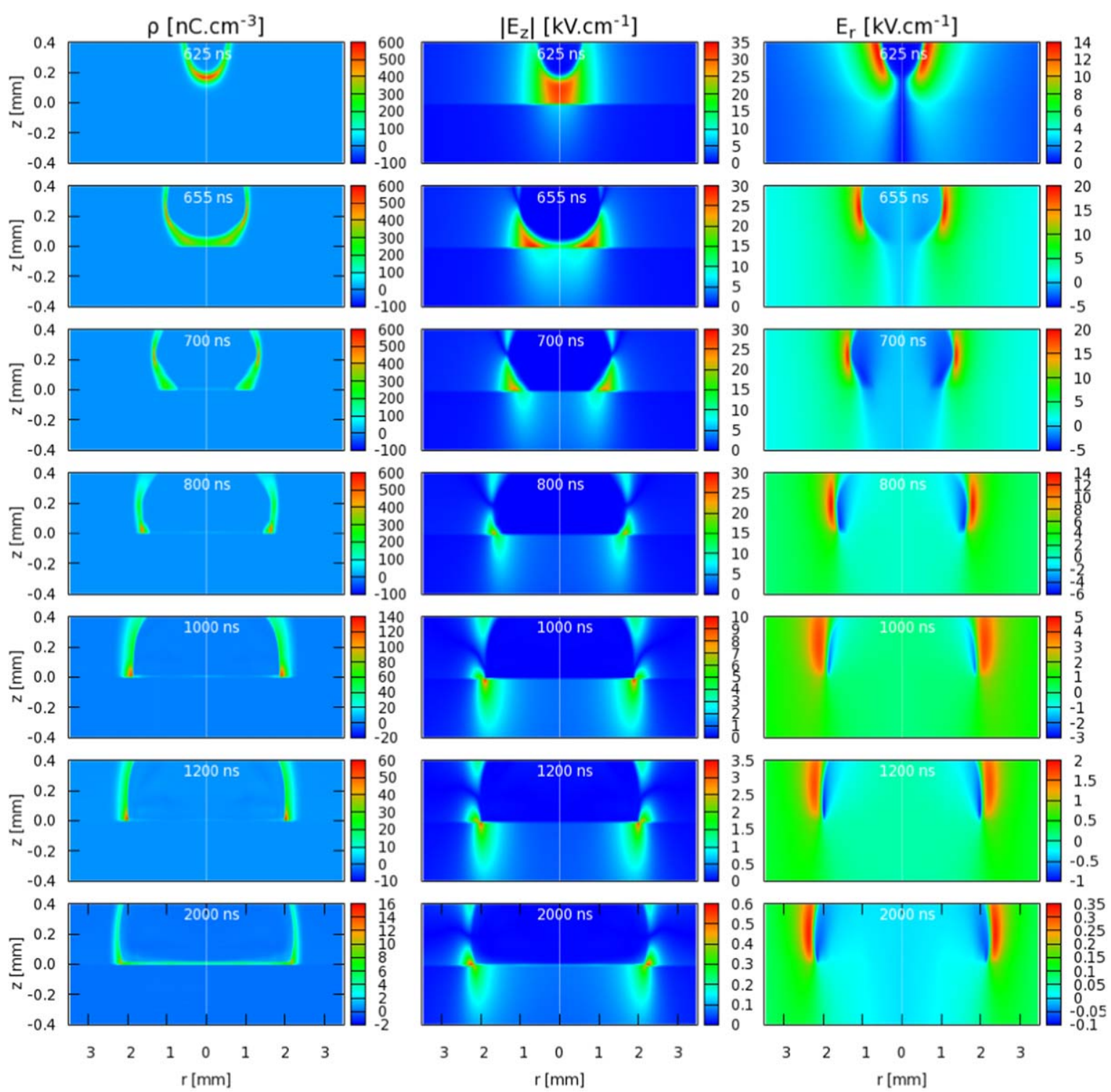

Figure 6. Cross sectional views of the volume charge density (left), of the magnitude of the axial (center) and radial (right) components of electric field during plasma-dielectric interaction at $t=625,655,700,800,1000,1200$ and $2000 \mathrm{~ns}$.

suggests that in a He-air mixture they would be mainly nitrogen and oxygen ions, as has been reported in Breden and Raja (2014), Ning et al (2018). It is also shown in figure 6 that $E_{z}$ is already present inside the target at $t=655 \mathrm{~ns}$, although with much lower intensity than in the plume, and that a radial component of electric field $E_{r}$ is also present, directed outwards both in the plume and inside the target. As the discharge is mechanically hampered from propagating downwards, this $E_{r}$ leads the positive discharge front to spread radially close to the target surface. Figure 6 also shows that at $t=655 \mathrm{~ns}$ the volume charge density $\rho$ and $E_{z}$ in the plasma plume are no longer centered, even though $\sigma$ has its peak at $r=0$. Then, between $t=655 \mathrm{~ns}$ and $t=800 \mathrm{~ns}$, we notice that $E_{z}$ and the peak of $\sigma$ follow the discharge spreading. At the center, the deposition of $\sigma$ first slows down and then $\sigma$ decreases. The decrease of $\sigma$ in this region is justified by an electric field reversal (explained later in the text) and therefore a deposition of negative charges that cancel part of the positive surface charges. The negative charges deposited through electric drift are dominantly electrons, that have a plasma mobility at least 50 times higher than negative ions. Furthermore, we notice that the peak value of $\sigma$ increases along the propagation on the surface during the pulse.

From the analysis of figure 6 it is visible that the values of the electric field components are very different in the plasma plume and inside the target. Moreover, the spatial distributions of $E_{z}$ and $E_{r}$ present each significant differences inside the target, not only radially but also axially, being higher close to the surface and lower farther from the surface. Then, at $t=800 \mathrm{~ns}$, the pulse of applied voltage starts decreasing and therefore the electric potential on the discharge front, connected to the powered electrode, decreases with it, which leads to a visible fast decrease of electric field and of $\rho$. However, we can notice that between $t=800 \mathrm{~ns}$ and the posterior moments there are still $E_{z}$ and $E_{r}$ present 


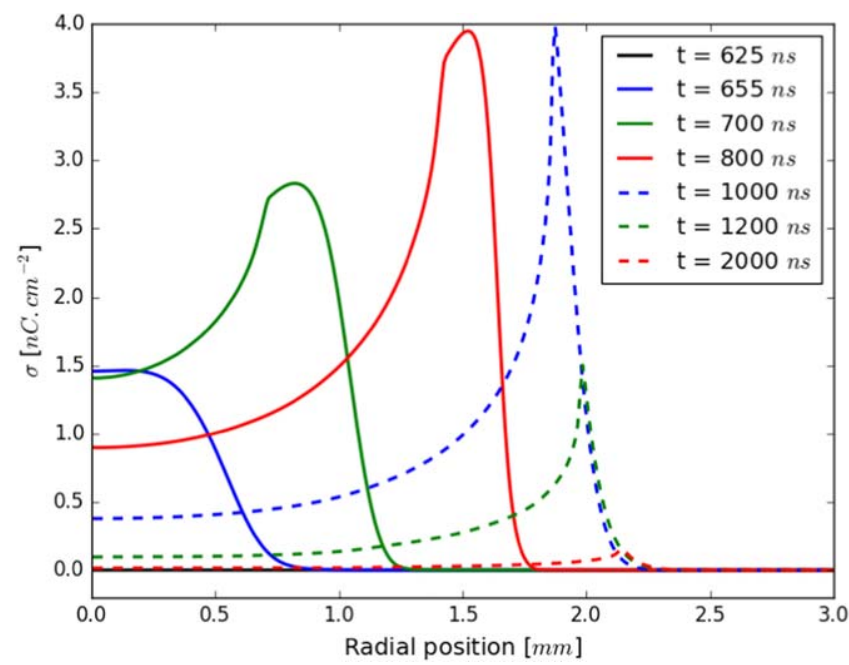

Figure 7. Radial profiles of the surface charge density deposited on the dielectric target surface at $t=625,655,700,800,1000,1200$ and $2000 \mathrm{~ns}$. Solid lines represent times during the pulse, while dashed lines refer to times after the pulse has ended.

inside the target, as well as there is still a slow radial spreading, due to the remaining values of $\rho$ and $E_{r}$. Concerning $\sigma$, figure 7 shows that after the end of the voltage pulse it slowly decreases to zero, which implies that in this phase $E_{z}$ on the surface creates a flux of deposition of negative charges. The decrease of $\sigma$ takes place until $t=1500 \mathrm{~ns}$. From that moment until $t=2000 \mathrm{~ns}$ the surface charge deposition has been confirmed to be approximately zero.

In order to obtain more detail into the dynamics between each type of charge and the electric field components, the separate contributions to the electric field calculation of $\rho$ in the plume exclusively and $\sigma$ on the target surface exclusively are assessed. These contributions are obtained by solving Poisson's equation (5), firstly with imposed applied voltage $V_{\text {app }}=0, \sigma=0$ and with $\rho$ taken from simulation results, and then with imposed $V_{\text {app }}=0, \rho=0$ and with $\sigma$ taken from simulation results, at every analyzed instant. It was also verified that the geometrical Laplacian contribution of electric field due to the applied voltage in the region of the dielectric target has a maximum value of $\sim 0.3 \mathrm{kV} \mathrm{cm}^{-1}$, which is negligible when compared to the other contributions during the plasma-dielectric interaction under study. Figure 8 presents the axial and radial components of electric field separately due to $\rho$ and $\sigma$, at the same moments and in the same region as figure 6 .

As the discharge arrives on the target surface at $t=625$ ns, figure 8 shows that the electric field in the plume and inside the target are firstly exclusively due to $\rho$, since no $\sigma$ is present on the surface. Then, at $t=655 \mathrm{~ns}$, as $\rho$ is not centered, the fields created by it $\left(E_{z \rho}\right.$ and $\left.E_{r \rho}\right)$ inside the target follow the same radial structure. However, we notice that $E_{z}$ created by $\sigma, E_{z \sigma}$, has its peak at $r=0$, corresponding to the radial profile of $\sigma$ present in figure 7 at $t=655 \mathrm{~ns}$. It is also noticeable that $E_{z \sigma}$ presents an almost symmetrical distribution in the region close to $r=0$, directed downwards inside the target and upwards in the plasma plume region. The jump of $E_{z}$ at the dielectric target interface with the plasma and the quasi-symmetrical distribution of $E_{z \sigma}$ are due to the permittivity difference between the dielectric and the plasma and to the surface charges deposited on the dielectric surface, in agreement with Gauss's law: $\left(\epsilon_{0} E_{z \rightarrow z^{+}}\right)-\left(\epsilon_{0} \epsilon_{r} E_{z \rightarrow z^{-}}\right)=\sigma$. This distribution implies that $E_{z}$ in the plasma plume in this region is the sum of opposite contributions. On the one hand, $\rho$ creates $E_{z}$ downwards, and on the other hand, $\sigma$ originates an upwards $E_{z}$. In fact, as positive charges deposit at the center of the target surface between $t=625 \mathrm{~ns}$ and $t=655$ ns, $\rho$ decreases, its maximum spreads radially and $\sigma$ increases at the center. This dynamics of charges leads to $E_{z}$ decreasing in value and then slightly reversing direction at $z=0$, leading to negative charge deposition, which explains the decrease of $\sigma$ at the center until $t=800 \mathrm{~ns}$ (figure 7). Then, a quasiequilibrium between both contributions to $E_{z}$ is reached and $E_{z}$ cancels at $r=0$. The electric field reversal after surface charge deposition on a dielectric target and the consequent opposite-polarity deposition have been reported in other works, such as Soloviev and Krivtsov (2009), Pechereau and Bourdon (2014). The same kind of evolution of $E_{z}$ takes place along the radial spreading on the target surface, behind the passage of the maximum of $\rho$. During the radial spreading of the discharge, between $t=655 \mathrm{~ns}$ and $t=800 \mathrm{~ns}$, we see that both electric field components, close to the surface and inside the target, are higher on the edge of the deposition region and result from both $\rho$ and $\sigma$ contributions. This coincides with the $\rho$ and $\sigma$ distributions in figures 6 and 7 . After $t=800 \mathrm{~ns}$, as the applied voltage decreases to zero, $\rho$ rapidly decreases and so do the electric field components originated by $\rho$. In particular, we notice that $E_{z \rho}$ that pushes positive charges towards the surface decreases. Then, at $z=0$, it is $E_{z \sigma}$ that dominates the interaction between charges in the plasma and the dielectric surface, leading to negative charge deposition, which decreases the values of positive $\sigma$, as observed in figure 7 . In this context of decrease of applied voltage, volume charges and surface charges, all the electric field components tend to very low values, as shown for $t=2000 \mathrm{~ns}$.

\section{Discussion on electric field measurements and simulations}

In this section, further analysis of the measured electric fields presented in section 4.1 is performed by complementing them with the numerical results from section 4.2.

The electric field in the target due to surface charges observed experimentally in figure 5 follows the behavior of the numerically predicted surface charging as a function of time, shown in figure 7 . In experiments, a temporal and radial distribution of the electric field inside the sample is observed. When the high voltage pulse ends, the electric field induced by surface charges disappears. Then, electric field of opposite sign is observed after the end of the pulse in the center of the impact region, suggesting that there are now negative surface charges. This reverse-polarity field is much smaller than the field during the pulse, indicating that the amount of opposite charge is significantly lower than the positive charge during 


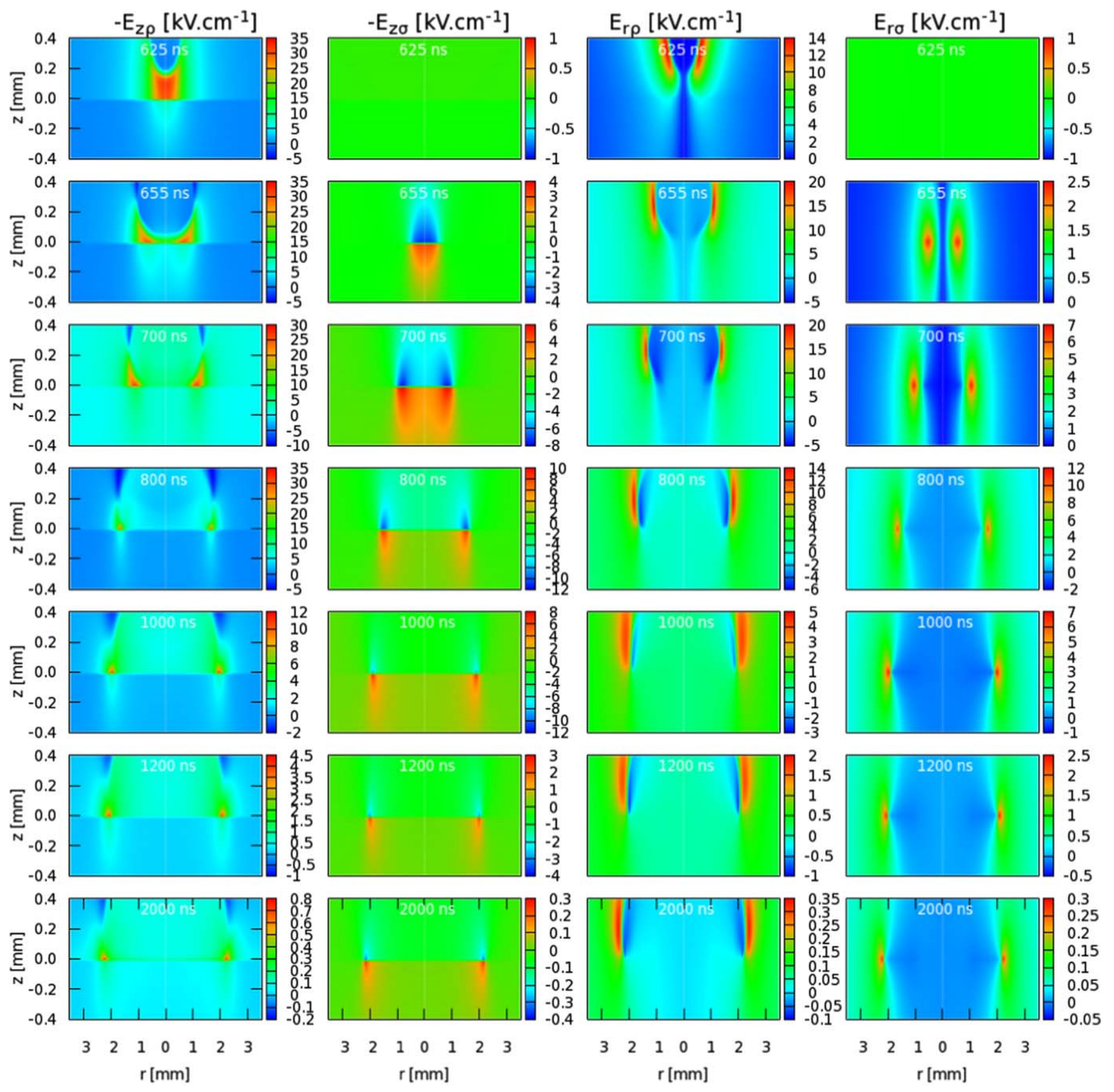

Figure 8. Cross sectional views of the $\rho$ and $\sigma$ contributions to $E_{z}$ and $E_{r}$ during plasma-dielectric interaction at $t=625,655,700,800,1000$, 1200 and $2000 \mathrm{~ns}$.

the pulse. We also notice in the experimental results that the axial electric field in the target spreads radially in time away from the impact region. Later, it indicates the location of the onset of anisotropic surface streamers. Such anisotropic phenomena cannot be described by an axisymmetric model, but the radial spreading of the axial electric field is observed as well in figures 6 and 8 .

The field values measured inside the dielectric are maximally $5 \mathrm{kV} \mathrm{cm}^{-1}$ in the axial direction and $3 \mathrm{kV} \mathrm{cm}^{-1}$ radially, and are lower than the one in the plasma plume reported in Sobota et al (2016). Using the numerical results presented in figures $6-8$, it is possible to provide further explanation on the lower values that are obtained inside the target. Its origin is important in order to understand which electric fields are generated inside dielectric targets that are exposed to plasma jets. The values obtained experimentally and presented in figure 5 are solely showing the contribution of surface charge density $\sigma$ to the field inside the dielectric, since the Pockels effect is exploited. These values are an average throughout the thickness of the material, due to the propagation of the polarized light that is used for the examination. Figure 9 shows how the averaged value of the axial component of electric field $E_{z}$ relates to the field on the surface in the simulation results. The radial profiles of $E_{z}$ are presented at $t=800 \mathrm{~ns}$, before the decrease of the applied voltage. The total $E_{z}$ is shown at the surface $(z=0)$, as well as 


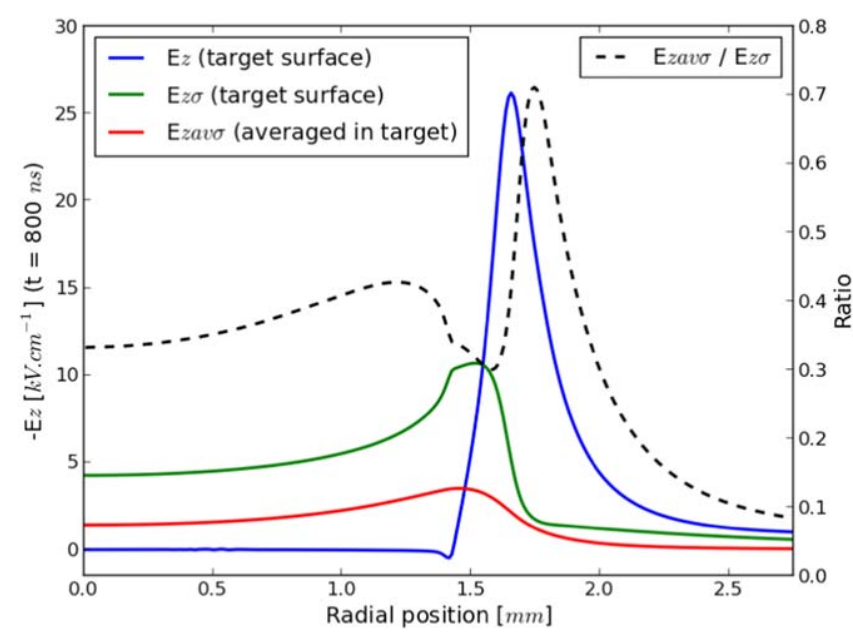

Figure 9. Radial profiles at the end of the pulse at $t=800 \mathrm{~ns}$. On the left-side vertical axis, $E_{z}$ on the target surface at $z=0.0 \mathrm{~mm}$ : total $E_{z}$ (blue line) and $E_{z \sigma}$ (green); and inside the target, averaged through its thickness: $E_{z a v \sigma}$ (red). On the right-side vertical axis, the ratio between the surface charge contributions averaged inside the target and on the target surface at $z=0.0 \mathrm{~mm}$.

the surface charge contribution $E_{z \sigma}$ at the surface and averaged through the thickness of the target. Additionally, the same figure presents the calculated ratio between $E_{z \sigma}$ averaged inside the target and on its surface.

The discharge front is close to the surface at approximately $r=1.8 \mathrm{~mm}$. The peak of the total $E_{z}$ is of $27 \mathrm{kV} \mathrm{cm}^{-1}$, and decreases rapidly to zero behind the discharge front. The difference between the profiles of electric field due to $\sigma$ on the surface and averaged inside the $0.5 \mathrm{~mm}$ thick target is clearly visible. It proves that the measured fields are inhomogeneous inside the target and surface charge densities cannot be estimated without any compensation. This has been pointed out already in Slikboer et al (2017). There, a procedure has been proposed to estimate the value of the axial electric field on the surface, due to $\sigma$, from the measured average value. The value on the surface can then be used to estimate the surface charge density. If no compensation is taken into account, an error on the estimation of the density values on the surface is introduced. The compensation procedure in Slikboer et al (2017) assumes a uniformly charged disk on the target surface and converts $E_{z \sigma}$ averaged in the target into its value on the surface through a ratio depending on the size of the charged disk and the thickness of the target. With the model, considering the full distribution of $\sigma$, we show that the uniformity of that distribution is also important to evaluate the compensation procedure. In fact, we see in figure 9 that the ratio between $E_{z \sigma}$ averaged in the target and on the surface is stable only in the region behind the discharge spreading, where the surface charges have already been deposited and have stabilized, and not in the region of the discharge front where $\sigma$ is going through fast variations. The ratio between $E_{z \sigma}$ averaged inside the target and on the target surface in the mentioned region is here presented with values between 0.3 and 0.4 .

Figure 10 shows the temporal evolution with ns resolution of the axial components of electric field $E_{z}$ at $r=0$ in the

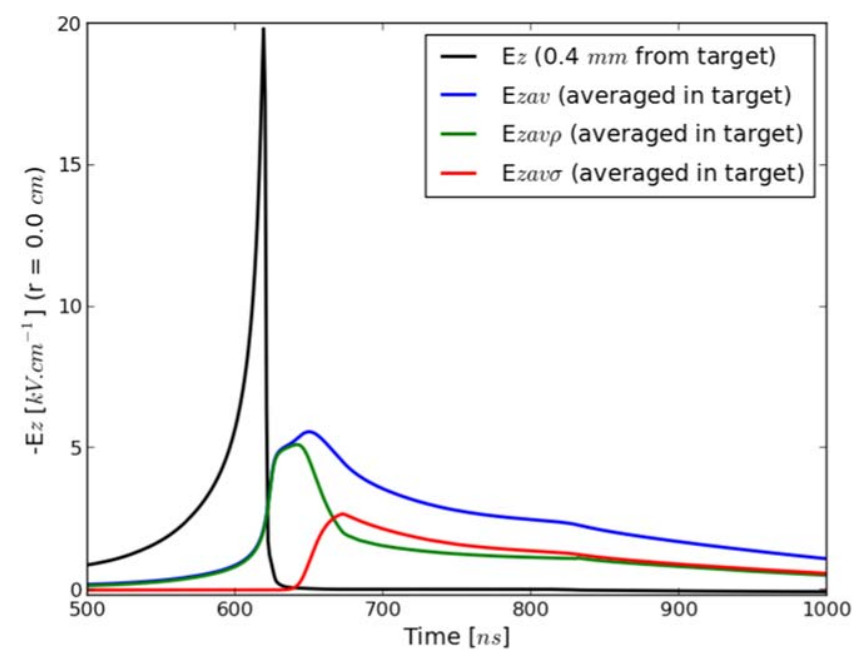

Figure 10. Temporal profiles of the axial component of electric field $E_{z}$ at $r=0.0 \mathrm{~mm}$ in the plasma plume at $z=0.4 \mathrm{~mm}$ (black line) and inside the dielectric target, averaged through its thickness: $E_{z a v}$ (blue), $E_{\text {zav } \rho}$ (green) and $E_{\text {zavo }}$ (red).

plasma plume and averaged inside the target through its $0.5 \mathrm{~mm}$ thickness. The temporal profile of the averaged field in the target is presented as total value and separated into the volume charge and surface charge contributions $E_{z \rho}$ and $E_{z \sigma}$. The black curve in figure 10 represents the temporal profile of $E_{z}$ very close to the target surface, at $0.4 \mathrm{~mm}$ distance. We notice that $E_{z}$ at this position increases as the volume charges in the discharge front approach the target and attains a maximum of around $20 \mathrm{kV} \mathrm{cm}^{-1}$. After the passage of $\rho$ at this spatial position, the electric field at that position cancels almost instantly. The peak value of $E_{z}$ in the plume is comparable to the one measured in Sobota et al (2016). The electric field in the plasma plume before the impact on the target is due only to volume charges in the discharge front, since there are still no charges deposited on the target surface. As such, at this point the volume charges dominate the propagation of the discharge. Inside the target, as shown in figure 10, the electric field values are lower, both before and during the plasma-surface interaction. The averaged $E_{z}$ inside the dielectric target is about 4 times lower than the value at $0.4 \mathrm{~mm}$ from the target. It has the same order of magnitude as the field measured experimentally with the (electro-optic) dielectric crystal, presented in figure 5. The field inside the dielectric target is due to both a contribution of $\rho$ and $\sigma$. By separating $E_{z}$ inside the target into $E_{z \rho}$ and $E_{z \sigma}$, we can distinguish the different influences on the electric field to which the target is exposed to. At the moment of discharge impact, $E_{z}$ inside the target is generated by the arrival of volume charges. Only later, as surface charges cumulate on the target surface, both $\rho$ and $\sigma$ provide contributions to the electric field inside the target at $r=0$. As the discharge spreads, we notice that both contributions decrease at $r=0$ to about $1.5 \mathrm{kV} \mathrm{cm}^{-1}$ each. Then, near $t=850 \mathrm{ns,}$, when the applied voltage becomes zero in the model, a decrease of $E_{z \rho}$ and $E_{z \sigma}$ takes place, leading to very small values of electric field, as had already been observed in figure 8 and experimentally in figure 5 . 


\section{Conclusions}

This work has investigated the plasma-target interaction occurring between a pulsed helium plasma jet and a dielectric target, both experimentally and numerically. Experiments and simulations have been performed with slightly different settings (electrode configuration, target permittivity and voltage pulse duration). However, in both cases, a centered discharge front impacts a dielectric surface and starts spreading on it. Then, in this work, we have carried out a phenomenological study with both experiments and simulations to better understand the role of the averaging of electric field over the dielectric thickness in the experiments and the contribution of surface and volume charges to the total electric field in the dielectric target.

Experimentally, the plasma-target interaction has been examined by measuring the electric field exclusively due to the surface charge density $\sigma$ inside an electro-optic target under exposure of the plasma jet. For the first time, the spatial distributions of both the axial and radial components of electric field inside the target due to $\sigma$ have been measured simultaneously, using Mueller polarimetry. The observed time-resolved dynamics during the plasma-target interaction shows a radial propagation of the electric field inside the dielectric as the plasma charges its surface during the pulse. Axial electric fields are measured up to $5 \mathrm{kV} \mathrm{cm}^{-1}$, located at the position of the maximum of surface charge density. Radial electric fields have lower magnitude, approximately $3 \mathrm{kV} \mathrm{cm}^{-1}$, but spread further. These electric field components experienced by the target are lower than generally reported for discharge propagation in the plasma plume.

Numerically, the investigation of the separate volume charge density $\rho$ and surface charge density $\sigma$ contributions to the electric field has allowed a detailed explanation of the dynamics of discharge propagation and plasma-target interaction, complementing the information obtained experimentally. We have pointed out the axial variation of electric field inside the dielectric target and the difference between the values of electric field on the target surface and averaged inside the target. Considering the non-uniform distribution of $\sigma$, it has been shown that the ratio between the axial component of electric field averaged inside the target and on the target surface has values between 0.3 and 0.4 in the region behind discharge propagation. Furthermore, it has been demonstrated that the axial component of electric field assessed in the discharge front before impact, exclusively due to $\rho$, has a peak value of $20 \mathrm{kV} \mathrm{cm}^{-1}$ at $0.4 \mathrm{~mm}$ from the target, while the same component of electric field averaged inside the dielectric target during the interaction is lower $(\sim 5$ $\mathrm{kV} \mathrm{cm}^{-1}$ ). These simulation results confirm the experimental finding that the electric field experienced by the target is lower than generally reported for discharge propagation. In fact, during the plasma-surface interaction, two effects sequentially determine the electric field inside the target: firstly, a relatively high electric field is observed due to the proximity of the ionization front and high volume charge density $\rho$; afterwards, in longer timescales, lower electric fields are induced due to the contribution of both leftover volume charges close to the target and surface charges deposited on its surface. The experimental technique of Mueller polarimetry provides a unique way to examine this second phase of the plasma-surface interaction.

This work has shown the interest to use, in a complementary way, space and time-resolved measurements and simulations of electric field components to better understand plasma-surface interactions that are crucial for many plasma applications. In a future work, we plan to carry out a detailed quantitative comparison of the values of the electric field inside the target measured in experiments and obtained in simulations for different flow rates and applied voltages.

\section{Acknowledgments}

PV and ES are supported by French governmental fellowships attributed by the École Doctorale Ondes et Matière. ES is also funded by the 'Chaire Énergie Durable' at École Polytechnique under the reference EXXI with the financial aid from EDF foundation. This work has been done partially within the LABEX Plas@par project, and received financial state aid managed by the Agence Nationale de la Recherche (ANR), as part of the programme 'Investissements d'avenir' under the reference ANR-11-IDEX-0004-02. AS would like to thank the ANR for the 'Invited International Expert' grant, hosted at École Polytechnique. ZB acknowledges support of project LO1411 (NPU I) funded by the Ministry of Education, Youth and Sports of the Czech Republic. Simulations presented in this work have been performed thanks to the computational resources of the clusters 'Hopper' at École Polytechnique and 'Zoidberg' at LPP.

\section{ORCID iDs}

Pedro Viegas (i) https://orcid.org/0000-0002-3820-3300 Elmar Slikboer (ib https://orcid.org/0000-0002-7716-907X Adam Obrusník (iD https://orcid.org/0000-0002-8415-4909 Zdenek Bonaventura (1) https://orcid.org/0000-00029591-6040

Ana Sobota (1) https://orcid.org/0000-0003-1036-4513

Olivier Guaitella (iD https://orcid.org/0000-0002-6509-6934

\section{References}

Allen N L and Mikropoulos P N 1999 Streamer propagation along insulating surfaces IEEE Trans. Dielectr. Electr. Insul. 6 357-62

Arjunan K P, Obrusník A, Jones B T, Zajickova L and Ptasinka S 2016 Effect of additive oxygen on the reactive species profile and microbicidal property of a helium atmospheric pressure plasma jet Plasma Process. Polym. 13 1089-105

Azzam R M A 1978 Propagation of partially polarized light through anisotropic media with or without depolarization: a differential $4 \times 4$ matrix calculus J. Opt. Soc. Am. 681756

Bogaczyk M, Wild R, Stollenwerk L and Wagner H-E 2012 Surface charge accumulation and discharge development in diffuse and 
filamentary barrier discharges operating in $\mathrm{He}, \mathrm{N}_{2}$ and mixtures J. Phys. D: Appl. Phys. 45465202

Bourdon A, Darny T, Pechereau F, Pouvesle J, Viegas P, Iséni S and Robert E 2016 Numerical and experimental study of the dynamics of a $\mu$ s helium plasma gun discharge with various amounts of $\mathrm{N}_{2}$ admixture Plasma Sources Sci. Technol. 25 035002

Breden D and Raja L L 2014 Computational study of the interaction of cold atmospheric helium plasma jets with surfaces Plasma Sources Sci. Technol. 23065020

Breden D, Miki K and Raja L L 2012 Self-consistent twodimensional modeling of cold atmospheric-pressure plasma jets/bullets Plasma Sources Sci. Technol. 21034011

Celestin S, Bonaventura Z, Guaitella O, Rousseau A and Bourdon A 2009 Influence of surface charges on the structure of a dielectric barrier discharge in air at atmospheric pressure: experiment and modeling Eur. Phys. J. Appl. Phys. 4722810

Cheng C, Liye Z and Zhan R-J 2006 Surface modification of polymer fibre by the new atmospheric pressure cold plasma jet Surf. Coat. Technol. 200 6659-65

Compain E, Poirier S and Drevillon B 1999 General and selfconsistent method for the calibration of polarization modulators, polarimeters, and Mueller-matrix ellipsometers Appl. Opt. 38 3490-502

COMSOL 2017 CFD module user's guide, version 5.3

Ehlbeck J, Schnabel U, Polak M, Winter J, Von Woedtke T, Brandenburg R, Von Dem Hagen T and Weltmann K D 2011 Low temperature atmospheric pressure plasma sources for microbial decontamination J. Phys. D: Appl. Phys. 44013002

Ellis H, Pai R, McDaniel E, Mason E and Viehland L 1976 Transport properties of gaseous ions over a wide energy range At. Data Nucl. Data Tables 17177

Fanelli F and Fracassi F 2017 Atmospheric pressure non-equilibrium plasma jet technology: general features, specificities and applications in surface processing of materials Surf. Coat. Technol. 322 174-201

Fridman A and Friedman G 2013 Plasma Medecine (New York: Wiley)

Fridman G, Friedman G, Gutsol A, Shekhter A B, Vasilets V N and Fridman A 2008 Applied plasmas medicine Plasma Process. Plym. 5 503-33

Garcia-Caurel E, Ossikovski R, Foldyna M, Drévillon B and Martino A D 2013 Ellipsometry at the Nanoscale (Berlin: Springer)

Gil J and Ossikovski R 2016 Polarized Light and the Mueller Matrix Approach (Boca Raton, FL: CRC Press)

Goldberg B M, Bohm P S, Czarnetzki U, Adamovich I V and Lempert W R 2015 Electric field vector measurements in a surface ionization wave discharge Plasma Sources Sci. Technol. 24055017

Graves D B 2012 The emerging role of reactive oxygen and nitrogen species in redox biology and some implications for plasma applications to medicine and biology J. Phys. D: Appl. Phys. 45263001

Guaitella O and Sobota A 2015 The impingement of a kHz helium atmospheric pressure plasma jet on a dielectric surface $J$. Phys. D: Appl. Phys. 48255202

Guaitella O, Thevenet F, Guillard C and Rousseau A 2006 Dynamic of the plasma current amplitude in a barrier discharge: influence of photocatalytic material J. Phys. D: Appl. Phys. 39 2964-72

Hagelaar G and Pitchford L 2005 Solving the Boltzmann equation to obtain electron transport coefficients and rate coefficients for fluid models Plasma Sources Sci. Technol. 14722

IST 2018 IST-Lisbon database, (Retrieved: January 2018)

Jánský J and Bourdon A 2014 Simulation of two counterpropagating helium discharges at atmospheric pressure Plasma Sources Sci. Technol. 23025001
Kawasaki T, Arai Y and Takada T 1991 Two-dimensional measurement of electrical surface charge distribution on insulating materials by electrooptic pockels effect Japan. $J$. Appl. Phys. 130 1262-5

Kong M G, Kroesen G, Morfill G, Nosenko T, Shimizu T, van Dijk J and Zimmermann J L 2009 Plasma medicine: an introductory review New J. Phys. 11115012

Kulikovsky A A 1997 Positive streamer between parallel plate electrodes in atmospheric pressure air J. Phys. D: Appl. Phys. 30441

Laroussi M and Leipold F 2004 Evaluation of the roles of reactive species, heat, and UV radiation in the inactivation of bacterial cells by air plasmas at atmospheric pressure Int. J. Mass Spectrom. 233 81-6

Lindsay A, Anderson C, Slikboer E, Shannon S and Graves D 2015 Momentum, heat, and neutral mass transport in convective atmospheric pressure plasma-liquid systems and implications for aqueous targets J. Phys. D: Appl. Phys. 48424007

Lindsay A, Byrns B, King W, Andhvarapou A, Fields J, Knappe D, Fonteno W and Shannon S 2014 Fertilization of radishes, tomatoes, and marigolds using a large-volume atmospheric glow discharge Plasma Chem. Plasma Process. 34 1271-90

Liu D, Rong M, Wang X, Iza F, Kong M and Bruggeman P 2010 Main species and physicochemical processes in cold atmospheric-pressure $\mathrm{He}+\mathrm{O}_{2}$ plasmas Plasma Process. Polym. 7846

Macías-Romero C and Török P 2012 Eigenvalue calibration methods for polarimetry J. Eur. Opt. Soc. 712004

Naidis G 2012 Modeling of helium plasma jets emerged into ambient air: influence of applied voltage, jet radius, and helium flow velocity on plasma jet characteristics J. Appl. Phys. 112 103304

Naidis G V 2011 Modelling of plasma bullet propagation along a helium jet in ambient air J. Phys. D: Appl. Phys. 44215203

Neyts E C 2016 Plasma-surface interactions in plasma catalysis Plasma Chem. Plasma Process. 36 185-212

Ning W, Dai D, Zhang Y, Han Y and Li L 2018 Effects of trace of nitrogen on the helium atmospheric pressure plasma jet interacting with a dielectric substrate J. Phys. D: Appl. Phys. 51125204

Noeske M, Degenhardt J, Strudthoff S and Lommatzsch U 2004 Plasma jet treatment of five polymers at atmospheric pressure: surface modifications and the relevance for adhesion Int. $J$. Adhes. Adhes. 24 171-7

Norberg S, Johnsen E and Kushner M 2015 Helium atmospheric pressure plasma jets touching dielectric and metal surfaces J. Appl. Phys. 118013301

Orlov D M, Font G I and Edelstein D 2008 Characterization of Discharge Modes of Plasma Actuators AIAA J. 46 3142-8

Ossikovski R 2011 Differential matrix formalism for depolarizing anisotropic media Opt. Lett. 36 2330-2

Pancheshnyi S, Biagi S, Bordage M, Hagelaar G, Morgan W, Phelps A and Pitchford L 2012 The LXCat project: Electron scattering cross sections and swarm parameters for low temperature plasma modeling Chem. Phys. 398148

Pechereau F 2013 Numerical simulation of the interaction of a plasma discharge at atmospheric pressure with dielectric surfaces $P h D$ Thesis Ecole Centrale Paris, France

Pechereau F and Bourdon A 2014 Influence of the polarity of the applied voltage on the reignition of a discharge below a dielectric layer in air at atmospheric pressure J. Phys. D: Appl. Phys. 47445206

Pechereau F, Jánský J and Bourdon A 2012 Simulation of the reignition of a discharge behind a dielectric layer in air at atmospheric pressure Plasma Sources Sci. Technol. 21055011

Sakiyama Y, Graves D B and Stoffels E 2008 Influence of electrical properties of treated surface on rf-excited plasma needle at atmospheric pressure J. Phys. D: Appl. Phys. 41095204 
Schmidt-Bleker A, Norberg S A, Winter J, Johnsen E, Reuter S, Weltmann K D and Kushner M J 2015 Propagation mechanisms of guided streamers in plasma jets: the influence of electronegativity of the surrounding gas Plasma Sources Sci. Technol. 24035022

Seo Y S, Mohamed A A H, Woo K C, Lee H W, Lee J K and Kim K T 2010 Comparative studies of atmospheric pressure plasma characteristics between $\mathrm{He}$ and Ar working gases for sterilization IEEE Trans. Plasma Sci. 38 2954-62

Slikboer E, Guaitella O and Sobota A 2016 Time-resolved electric field measurements during and after the initialization of a $\mathrm{kHz}$ plasma jet - from streamers to guided streamers Plasma Sources Sci. Technol. 25 03LT04

Slikboer E, Garcia-Caurel E, Guaitella O and Sobota A 2017 Charge transfer to a dielectric target by guided ionization waves using electric field measurements Plasma Sources Sci. Technol. 26 035002

Slikboer E, Sobota A, Guaitella O and Garcia-Caurel E 2018a Electric field and temperature in a target induced by a plasma jet imaged using Mueller polarimetry J. Phys. D: Appl. Phys. 51025204

Slikboer E, Sobota A, Guaitella O and Garcia-Caurel E 2018b Imaging axial and radial electric field components in dielectric targets under plasma exposure J. Phys. D: Appl. Phys. 51 115203

Sobota A, Guaitella O and Garcia-Caurel E 2013 Experimentally obtained values of electric field of an atmospheric pressure plasma jet impinging on a dielectric surface J. Phys. D: Appl. Phys. 46372001

Sobota A, Guaitella O, Sretenovic G, Krstic I, Kovacevic V, Obrusník A, Nguyen Y, Zajickova L, Obradovic B and Kuraica M 2016 Electric field measurements in a kHz-driven He jet - the influence of the gas flow speed Plasma Sources Sci. Technol. 25065026

Sobota A, Lebouvier A, Kramer N J, van Veldhuizen E M, Stoffels W W, Manders F and Haverlag M 2009 Speed of streamers in argon over a flat surface of a dielectric J. Phys. D: Appl. Phys. 42015211

Soloviev V R and Krivtsov V M 2009 Surface barrier discharge modelling for aerodynamic applications J. Phys. D: Appl. Phys. 42125208

Stollenwerk L, Laven J G and Purwins H-G 2007 Spatially resolved surface-charge measurement in a planar dielectric-barrier discharge system Phys. Rev. Lett. 98255001
Tanaka H and Hori M 2017 Medical applications of non-thermal atmospheric pressure plasma J. Clin. Biochem. Nutrition 60 29-32

Tian W and Kushner M J 2014 Atmospheric pressure dielectric barrier discharges interacting with liquid covered tissue J. Phys. D: Appl. Phys. 47165201

Tschiersch R, Bogaczyk M and Wagner H E 2014 Systematic investigation of the barrier discharge operation in helium, nitrogen, and mixtures: discharge development, formation and decay of surface charges J. Phys. D: Appl. Phys. 4736

Unfer T and Boeuf J P 2009 Modelling of a nanosecond surface discharge actuator J. Phys. D: Appl. Phys. 4219

Viegas P, Pechereau F and Bourdon A 2018 Numerical study on the time evolutions of the electric field in helium plasma jets with positive and negative polarities Plasma Sources Sci. Technol. 27025007

Vorác J, Obrusník A, Procházka V, Dvorak P and Talába M 2014 Spatially resolved measurement of hydroxyl radical $(\mathrm{OH})$ concentration in an argon RF plasma jet by planar laserinduced fluorescence Plasma Sources Sci. Technol. 23025011

Wang L, Zheng Y and Jia S 2016 Numerical study of the interaction of a helium atmospheric pressure plasma jet with a dielectric material Phys. Plasmas 23103504

Weltmann K-D, Kindel E, Brandenburg R, Meyer C, Bussiahn R, Wilke C and von Woedtke T 2009 Atmospheric pressure plasma jet for medical therapy: plasma parameters and risk estimation Contrib. Plasma Phys. 49 631-40

Weltmann K-D and von Woedtke T 2017 Plasma medicine-current state of research and medical application Plasma Phys. Control. Fusion 59014031

Wild R, Gerling T, Bussiahn R, Weltmann K-D and Stollenwerk L 2014 Phase-resolved measurement of electric charge deposited by an atmospheric pressure plasma jet on a dielectric surface J. Phys. D: Appl. Phys. 47042001

Winter J, Sousa J S, Sadeghi N, Schmidt-Bleker A, Reuter S and Puech V 2015 The spatio-temporal distribution of $\mathrm{He}\left(2^{3} \mathrm{~S}_{1}\right)$ metastable atoms in a Mhz-driven helium plasma jet is influenced by the oxygen/nitrogen ratio of the surrounding atmosphere Plasma Sources Sci. Technol. 24025015

Zhu Y, Takada T and Tu D 1995 An optical measurement technique for studying residual surface charge distribution J. Phys. D: Appl. Phys. 28 1468-77 